\title{
Erratum: Novel construction and the monodromy relation for three-point functions at weak coupling
}

\author{
Yoichi Kazama, ${ }^{a}$ Shota Komatsu ${ }^{b}$ and Takuya Nishimura ${ }^{a}$ \\ ${ }^{a}$ Institute of Physics, University of Tokyo, \\ Komaba, Meguro-ku, Tokyo 153-8902 Japan \\ ${ }^{b}$ Perimeter Institute for Theoretical Physics, \\ Waterloo, Ontario N2L 2Y5, Canada \\ E-mail: kazama@hep1.c.u-tokyo.ac.jp, skomatsu@perimeterinstitute.ca, \\ tnishimura@hep1.c.u-tokyo.ac.jp
}

ERratum to: JHEP01(2015)095

\section{ARXIV EPRINT: 1410.8533}

In figure 6 on page 23, the identical figure is inadvertently used for all (a), (b) and (c). The correct figures are the ones shown below:

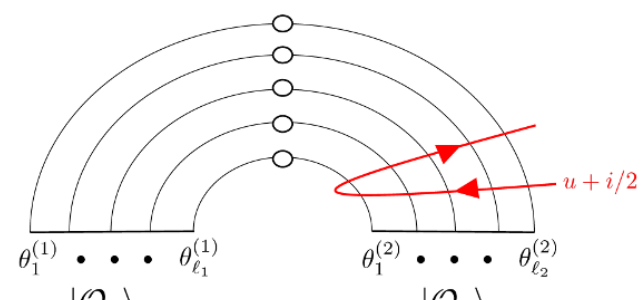

$\left|\mathcal{O}_{1}\right\rangle_{L}$
$\left|\mathcal{O}_{2}\right\rangle_{L}$

(a)

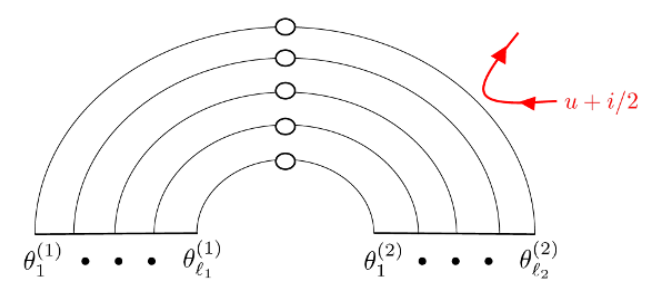

$\left|\mathcal{O}_{1}\right\rangle_{L}$
$\left|\mathcal{O}_{2}\right\rangle_{L}$

(b)

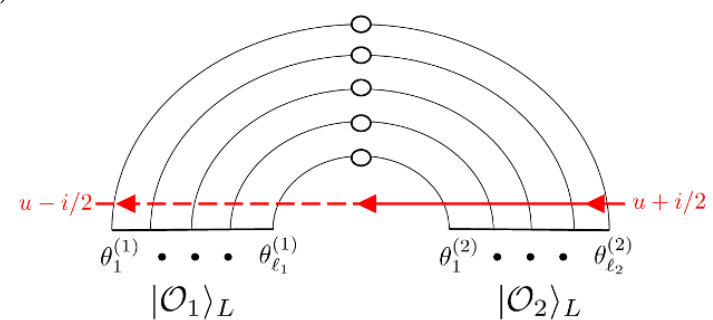

(c)

Open Access. This article is distributed under the terms of the Creative Commons Attribution License (CC-BY 4.0), which permits any use, distribution and reproduction in any medium, provided the original author(s) and source are credited. 